\title{
Lorentz-covariant, unitary evolution of a relativistic Majorana qubit
}

\author{
Jakub Rembieliński ${ }^{1}$ Paweł Caban ${ }^{1}$ (D) Kordian A. Smoliński ${ }^{1}$. \\ Sviatoslav Khrapko ${ }^{2}$
}

Received: 11 April 2018 / Accepted: 8 August 2018 / Published online: 23 August 2018

(c) The Author(s) 2018

\begin{abstract}
We formulate a covariant description of a relativistic qubit identified with an irreducible set of quantum spin states of a Majorana particle with a sharp momentum. We treat the particle's four-momentum as an external parameter. We show that it is possible to define an interesting time evolution of the spin density matrix of such a qubit. This evolution is manifestly Lorentz covariant in the bispinor representation and unitary in the spin representation. Moreover, during this evolution the Majorana particle undergoes an uniformly accelerated motion. We classify possible types of such motions, and finally we illustrate the behaviour of the polarization vector of the Majorana qubit during the evolution in some special cases.
\end{abstract}

Keywords Relativistic qubit - Relativistic quantum information theory · Lorentz-covariant evolution

CR Subject Classification Relativistic quantum information

\section{Introduction}

In the last two decades, the relativistic quantum information theory became an important area of research, see, e.g. [1,2,5,7-10,12,15,19,21,23-27]. One of the difficulties

\footnotetext{
$\bowtie \quad$ Paweł Caban

p.caban@merlin.phys.uni.lodz.pl

Jakub Rembieliński

jaremb@uni.lodz.pl

Kordian A. Smoliński

k.a.smolinski@merlin.phys.uni.lodz.pl

1 Department of Theoretical Physics, Faculty of Physics and Applied Informatics, University of Lodz, Pomorska 149/153, 90-236 Lodz, Poland

2 Yuriy Fedkovych Chernivtsi National University, Chernivtsi, Ukraine
} 
in this theory is related to the notion of a qubit modelled on the space of states of a massive spinning relativistic particle. The main problem lies in the fact that the procedure leading to the reduced density matrix is not Lorentz covariant regardless of the Lorentz invariance of the integration measure [22] (see also [16]). This problem stems from the fact that the Wigner rotation is momentum-dependent and consequently wave packets with momentum spread transforms differently for each energy/momentum mode. This problem was overcame in our previous paper [11] where we obtained the Lorentz-covariant reduced matrix which contains not only the information about the polarization of the particle but also the information about average values of the kinematical degrees of freedom. However, because the averages do not keep of the physically important interrelations like dispersion relation for four-momentum, Minkowski transversality condition between four-momentum and relativistic pseudospin four-vector, etc., we restrict here our attention to the case of sharp momentum treated as an external parameter. Therefore, in the present paper we define a covariant form of a spin density matrix in the bispinor basis for a relativistic Majorana particle with a sharp four-momentum. We choose the Majorana particle as an elementary system since it is the simplest spin-1/2 relativistic system. (In contrast, Dirac field admits particle and antiparticle excitations.) However, the main goal of this paper is to propose a model of a manifestly Lorentz- covariant, unitary evolution of a qubit. Unitarity of the evolution enables us to treat four-momentum as an external classical parameter-in this sense, our approach is a semiclassical one. On the other hand, the spin state of the particle undergoes an unitary evolution. This approach is similar to that used in the description of kinematical evolution of particles in SternGerlach experiment. Therefore, an interpretation of four-momentum as an external parameter allows to associate the classical trajectory with the motion of the Majorana particle. We consider the class of motions for which the square of a four-acceleration is constant during the evolution.

The proposed model of evolution can be useful in the discussion of spin correlations and entanglement evolution in experiments with accelerating particles. Paper on this subject is in preparation.

\section{The setting}

In this section, we introduce basic notions, definitions, and conventions used through this paper.

\subsection{Majorana field}

We recall here briefly the description of Majorana spin 1/2 particles.

The Majorana field operator has the standard momentum expansion

$$
\hat{\phi}_{\alpha}(x)=\frac{1}{(2 \pi)^{3 / 2}} \sum_{\sigma= \pm 1 / 2} \int \frac{d^{3} \mathbf{k}}{2 k^{0}}\left[e^{-i k x} u_{\alpha \sigma}(k) a_{\sigma}(k)+e^{i k x} v_{\alpha \sigma}(k) a_{\sigma}^{\dagger}(k)\right]
$$


where $\alpha$ is a bispinor index, $a_{\sigma}^{\dagger}(k)$ are creation operators of the particle with fourmomentum $k$, mass $m$, and spin component along the $z$-axis equal to $\sigma$; moreover, $k^{0}=$ $\sqrt{\mathbf{k}^{2}+m^{2}}$. Creation and annihilation operators fulfil the canonical anticommutation relations

$$
\left\{a_{\sigma^{\prime}}(k), a_{\sigma}^{\dagger}(p)\right\}=2 k^{0} \delta^{3}(\mathbf{k}-\mathbf{p}) \delta_{\sigma \sigma^{\prime}}
$$

The field operator, $\hat{\phi}(x)$, fulfils the Dirac equation

$$
\left(i \gamma^{\mu} \partial_{\mu}-m I\right) \hat{\phi}(x)=0
$$

where $\gamma^{\mu}$ are Dirac matrices (for our convention concerning Dirac matrices, see "Appendix A"). This equation implies the following standard conditions for amplitudes:

$$
\begin{aligned}
& (k \gamma+m I) v(k)=0, \\
& (k \gamma-m I) u(k)=0,
\end{aligned}
$$

where $v(k)$ and $u(k)$ denote matrices $\left[v_{\alpha \sigma}(k)\right]$ and $\left[u_{\alpha \sigma}(k)\right]$, respectively, and $k \gamma=$ $k_{\mu} \gamma^{\mu}$. Operators $v(k)$ and $u(k)$ can be treated as intertwining operators between spin basis (corresponding to indices $\sigma$ ) and covariant bispinor basis (corresponding to indices $\alpha$ ).

For further convenience, let us introduce the standard projectors

$$
\Lambda_{ \pm}(k)=\frac{m I \pm k \gamma}{2 m}
$$

Next, the field operator transforms under Lorentz group action in the following way:

$$
U(\Lambda) \hat{\phi}(x) U^{\dagger}(\Lambda)=S^{-1}(\Lambda) \hat{\phi}(\Lambda x),
$$

where $U(\Lambda)$ is an element of the unitary irreducible representation of the Poincare group and $S(\Lambda)$ is the bispinor representation of the Lorentz group (for the explicit form of bispinor representation employed in this paper, see "Appendix A", in particular $S(\Lambda)$ is given in Eq. (78)).

Creation and annihilation operators transform as follows:

$$
\begin{aligned}
& U(\Lambda) a_{\sigma}(k) U^{\dagger}(\Lambda)=\mathbb{W}^{\dagger}(\Lambda, k)_{\sigma \lambda} a_{\lambda}(\Lambda k), \\
& U(\Lambda) a_{\sigma}^{\dagger}(k) U^{\dagger}(\Lambda)=\mathbb{W}^{T}(\Lambda, k)_{\sigma \lambda} a_{\lambda}^{\dagger}(\Lambda k),
\end{aligned}
$$

where $\mathbb{W}(\Lambda, k) \in \mathrm{SL}(2, \mathbb{C})$ corresponds to the Wigner rotation in a standard homomorphism of $\mathrm{SL}(2, \mathbb{C})$ onto a Lorentz group, compare (79).

Consistency of Eqs. (6 and 7) imply standard Weinberg conditions for amplitudes

$$
\begin{aligned}
& v(\Lambda k)=S(\Lambda) v(k) \mathbb{W}^{T}(\Lambda, k), \\
& u(\Lambda k)=S(\Lambda) u(k) \mathbb{W}^{\dagger}(\Lambda, k) .
\end{aligned}
$$


A Majorana particle is its own antiparticle; therefore, it does not change under the action of the charge conjugation operator, i.e.

$$
\mathrm{C} a_{\sigma}^{\dagger}(k) C^{\dagger}=a_{\sigma}^{\dagger}(k)
$$

where $C$ is a charge conjugation operator.

The charge conjugation operator acting on the Majorana field gives

$$
\mathrm{C} \hat{\phi}(x) \mathrm{C}^{\dagger}=\mathscr{C}_{\hat{\phi}^{T}}(x)=\hat{\phi}(x)
$$

where we use standard notation $\hat{\bar{\phi}}=\hat{\phi}^{\dagger} \gamma^{0}$ and $\mathscr{C}$ is the charge conjugation matrix (75). Equation (10) implies the following condition for amplitudes:

$$
\begin{aligned}
& u(k)=\mathscr{C} \bar{v}^{T}(k), \\
& v(k)=\mathscr{C} \bar{u}^{T}(k) .
\end{aligned}
$$

Explicit form of amplitudes depends on the chosen representation of gamma matrices. We have determined $v(k)$ in "Appendix A"; its final form is the following:

$$
v(k)=\frac{1}{\sqrt{2}}\left(\begin{array}{c}
\mathbb{L}_{k} \\
-\mathbb{L}_{k}^{-1}
\end{array}\right) \sigma_{2}=\frac{1}{2 \sqrt{1+\frac{k^{0}}{m}}}\left(\begin{array}{c}
\left(I+\frac{1}{m} k^{\mu} \sigma_{\mu}\right) \sigma_{2} \\
-\left(I+\frac{1}{m} k^{\pi \mu} \sigma_{\mu}\right) \sigma_{2}
\end{array}\right),
$$

where $k^{\pi}=\left(k^{0},-\mathbf{k}\right)$, and $\sigma_{i}, i=1,2,3$, are the standard Pauli matrices, $\sigma_{0}=I$. The explicit form of the amplitude $u(k)$ can be found with the help of Eq. (11a)

$$
u(k)=i \gamma^{5} v(k) \sigma_{2}=\frac{i}{\sqrt{2}}\left(\begin{array}{l}
\mathbb{L}_{k} \\
\mathbb{L}_{k}^{-1}
\end{array}\right)
$$

where $\mathbb{L}_{k}$ is the matrix corresponding to the standard Lorentz boost transforming the particle of the mass $m$ from the rest to the state with four-momentum $k$ (see "Appendix A", Eq. (80) for the details).

\subsection{One-particle states}

The state of a particle with momentum $k$ and spin component $\sigma$ in the spin basis is defined as

$$
|k, \sigma\rangle=a_{\sigma}^{\dagger}(k)|0\rangle,
$$

where $|0\rangle$ is a Lorentz-invariant vacuum vector, $\langle 0 \mid 0\rangle=1, a_{\sigma}(k)|0\rangle=0$.

The states $\{|k, \sigma\rangle\}$ span the carrier space of the irreducible unitary representation $U(\Lambda)$ of the Poincaré group

$$
U(\Lambda)|k, \sigma\rangle=\mathbb{W}(\Lambda, k)_{\lambda \sigma}|\Lambda k, \lambda\rangle,
$$


where $\mathbb{W}(\Lambda, k)$ is defined in (7). Equation (2) implies that states $\{|k, \sigma\rangle\}$ are normalized covariantly

$$
\left\langle k, \sigma \mid k^{\prime}, \sigma^{\prime}\right\rangle=2 k^{0} \delta^{3}\left(\mathbf{k}-\mathbf{k}^{\prime}\right) \delta_{\sigma \sigma^{\prime}}
$$

where $k=\left(k^{0}, \mathbf{k}\right)$. Notice that Eq. (15) is consistent with Eq. (6) due to Weinberg conditions (8).

With the help of operators $v(k)$, one can define states ${ }^{1}$

$$
|\alpha, k\rangle=v_{\alpha \sigma}(k)|k, \sigma\rangle
$$

transforming covariantly under Lorentz group action

$$
U(\Lambda)|\alpha, k\rangle=S^{-1}(\Lambda)_{\alpha \beta}|\beta, \Lambda k\rangle
$$

Here $S(\Lambda)$ is a bispinor representation of a Lorentz transformation; its explicit form is given in Eq. (78). Therefore, (17) defines an overcomplete bispinor basis in a Hilbert space of one-particle states. It holds

$$
\bar{S}(\Lambda)=\gamma^{0} S^{\dagger}(\Lambda) \gamma^{0}=S^{-1}(\Lambda)
$$

Using Eq. (84), we find

$$
|k, \sigma\rangle=-\bar{v}(k)_{\sigma \alpha}|\alpha, k\rangle
$$

where $\bar{v}(k)=v(k)^{\dagger} \gamma_{0}$.

\section{Relativistic Majorana qubit}

The problem of a covariant reduced spin density matrix in a relativistic framework was widely discussed in the literature, see, e.g. [11,16,22].

Here we restrict our attention to a particle with a definite (sharp) momentum. The density operator describing such a particle can be written in the spin basis $\{|k, \sigma\rangle\}$ as

$$
\hat{\rho}(k)=\rho(k)_{\sigma \lambda}|k, \sigma\rangle\langle k, \lambda|
$$

The same density operator can be written in the covariant basis $\{|\alpha, k\rangle\}$ as

$$
\hat{\rho}(k)=\Omega(k)_{\beta \alpha}|\alpha, k\rangle \overline{\langle\beta, k|},
$$

(notice the reversed order of indices in $\Omega$, we use such an order for further convenience) where we define

$$
\overline{\langle\alpha, k|} \equiv\langle\beta, k| \gamma_{\beta, \alpha}^{0}=\langle k, \sigma| \bar{v}(k)_{\sigma \alpha}
$$

\footnotetext{
1 Notice our notational convention: covariant vectors (17) and standard basis vectors (14) differ by the order of arguments.
} 
Taking into account Eq. (17), matrices $\rho(k)=\left[\rho(k)_{\sigma \lambda}\right]$ and $\Omega(k)=\left[\Omega(k)_{\alpha \beta}\right]$ can be related via the following equation:

$$
\rho^{T}(k)=\bar{v}(k) \Omega(k) v(k) .
$$

To determine $\Omega(k)$ as a function of $\rho(k)$, we have to take into account that vectors $|\alpha, k\rangle$ are not linearly independent. Therefore, to fix uniquely a matrix $\Omega$, we demand that

$$
\Lambda_{-} \Omega(k) \Lambda_{-}=\Omega(k)
$$

Equations (24 and 25) give

$$
\Omega(k)=v(k) \rho^{T}(k) \bar{v}(k) .
$$

Now, applying Lorentz transformation $U(\Lambda)$ to the density operator $\hat{\rho}(k)$ we get

$$
\begin{aligned}
U(\Lambda) \hat{\rho}(k) U^{\dagger}(\Lambda) & =\rho^{\prime}(\Lambda k)_{\sigma \lambda}|\Lambda k, \sigma\rangle\langle\Lambda k, \lambda| \\
& =\Omega^{\prime}(\Lambda k)_{\beta \alpha}|\alpha, \Lambda k\rangle \overline{\langle\beta, \Lambda k|}
\end{aligned}
$$

and using Eqs. (15 and 18) we find the following transformation rules for matrices $\rho(k)$ and $\Omega(k)$

$$
\begin{aligned}
& \rho^{\prime}(\Lambda k)=\mathbb{W}(\Lambda, k) \rho(k) \mathbb{W}^{\dagger}(\Lambda, k), \\
& \Omega^{\prime}(\Lambda k)=S(\Lambda) \Omega(k) S^{-1}(\Lambda) .
\end{aligned}
$$

We see that, in contrast to the density matrix $\rho$, the matrix $\Omega(k)$ transforms in a manifestly covariant way under Lorentz group action.

The properties of the matrix $\rho(k)$ imply that it can be written in the standard Bloch form

$$
\rho(k)=\frac{1}{2}(I+\xi(k) \cdot \boldsymbol{\sigma}), \quad|\boldsymbol{\xi}| \leq 1
$$

The corresponding matrix $\Omega(k)$ can be determined with the help of Eq. (26). We obtain

$$
\Omega(k)=\frac{1}{4}\left(\begin{array}{c|c}
-I-\frac{2}{m^{2}}(k \sigma)\left(w^{\pi} \sigma\right) & \frac{1}{m}((k \sigma)-2(w \sigma)) \\
\hline \frac{1}{m}\left(\left(k^{\pi} \sigma\right)+2\left(w^{\pi} \sigma\right)\right) & -I+\frac{2}{m^{2}}\left(k^{\pi} \sigma\right)(w \sigma)
\end{array}\right),
$$

where we introduced the Pauli-Lubanski four-vector

$$
w^{0}=\frac{\mathbf{k} \cdot \boldsymbol{\xi}}{2}, \quad \mathbf{w}=\frac{1}{2}\left(m \boldsymbol{\xi}+\frac{\mathbf{k}(\mathbf{k} \cdot \boldsymbol{\xi})}{m+k^{0}}\right)
$$

satisfying:

$$
L_{k}\left(\begin{array}{l}
0 \\
\xi
\end{array}\right)=\frac{2}{m} w, \quad k w=0, \quad w^{2}=-\frac{1}{4} m^{2} \xi^{2}
$$


$L_{k}$ stands here for the standard boost defined before Eq. (80). We also use the standard notation $k^{\pi}=\left(k^{0},-\mathbf{k}\right), k \sigma=k^{\mu} \sigma_{\mu}$ (and analogously $w^{\pi}=\left(w^{0},-\mathbf{w}\right)$ and $w \sigma=$ $\left.w^{\mu} \sigma_{\mu}\right)$.

In terms of gamma matrices, the density matrix $\Omega(k)$ takes the form

$$
\Omega(k)=\frac{1}{4}\left(\frac{k \gamma}{m}-I\right)\left(I+2 \gamma^{5} \frac{w \gamma}{m}\right) .
$$

Moreover, the relation $k w=0$ implies that $(k \gamma)(w \gamma)=-(w \gamma)(k \gamma)$.

Notice that the hermicity of $\rho(k)$ implies

$$
\bar{\Omega}(k)=\Omega(k),
$$

while the property $\operatorname{Tr} \rho(k)=1$ gives

$$
\operatorname{Tr} \Omega(k)=-1
$$

From Eq. (35), we see that the covariant matrix $\Omega(k)$, representing the density matrix $\rho$ in the bispinor basis, is a function of $k$ and $\xi$ (via the Pauli-Lubanski four-vector). In the following, we will treat four-momentum as an external parameter determining a classical kinematical state of a particle. The quantum state of a particle is determined by spin.

\subsection{Spin observable}

The spin operator in the spin basis is defined as

$$
\hat{\mathbf{S}}=\frac{\sigma}{2}
$$

Thus, to calculate the average value of the spin observable in a state $\hat{\rho(k)}$ we use the formula

$$
\langle\mathbf{S}\rangle=\operatorname{Tr}\left(\rho(k) \frac{\boldsymbol{\sigma}}{2}\right) .
$$

Therefore, using $(39,24,25)$ and taking into account that $\Lambda_{-} u(k)=0$ we find

$$
\langle\mathbf{S}\rangle=\operatorname{Tr}\left(\Omega(k) \frac{v(k) \boldsymbol{\sigma}^{T} \bar{v}(k)}{2}\right)=\operatorname{Tr}\left[\Omega(k)\left(\frac{v(k) \boldsymbol{\sigma}^{T} \bar{v}(k)}{2}+\frac{u(k) \boldsymbol{\sigma}^{T} \bar{u}(k)}{2}\right)\right] .
$$

We have included terms with $u(k)$ to be able to apply the spin operator to negative energy states. So, finally, in the bispinor basis the spin operator takes the following form:

$$
\hat{\mathbf{S}}=\frac{v(k) \sigma^{T} \bar{v}(k)}{2}+\frac{u(k) \sigma^{T} \bar{u}(k)}{2} .
$$


Explicitly, we have:

$$
\hat{\mathbf{S}}=\frac{1}{2 m}\left[P^{0}\left(\begin{array}{ll}
\boldsymbol{\sigma} & 0 \\
0 & \boldsymbol{\sigma}
\end{array}\right)-i \mathbf{P} \times\left(\begin{array}{cc}
\boldsymbol{\sigma} & 0 \\
0 & -\boldsymbol{\sigma}
\end{array}\right)-\frac{\mathbf{P}}{m+P^{0}}\left(\begin{array}{cc}
\mathbf{P} \cdot \boldsymbol{\sigma} & 0 \\
0 & \mathbf{P} \cdot \boldsymbol{\sigma}
\end{array}\right)\right]
$$

It is worth noticing here that the problem of choice of a proper spin observable for a relativistic particle has a long history, see, e.g. [4,6,14] and references therein. In our previous papers, e.g. $[13,14]$, we have shown that the best spin operator has the following form:

$$
\hat{\mathbf{S}}_{N W}=\frac{1}{m}\left(\hat{\mathbf{W}}-\hat{W}^{0} \frac{\mathbf{P}}{m+P^{0}}\right),
$$

where

$$
\hat{W}^{\mu}=-\frac{1}{2} \varepsilon^{\mu \nu \mu^{\prime} v^{\prime}} P_{\nu} \hat{J}_{\mu^{\prime} v^{\prime}}
$$

is a Pauli-Lubanski four-vector and $\hat{J}_{\mu \nu}$ are generators of the Lorentz group, in a bispinor case

$$
\hat{J}_{\mu \nu}=\frac{i}{4}\left[\gamma_{\mu}, \gamma_{\nu}\right]
$$

In Eq. (43), we used the subscript ' $N W$ ' because this spin operator is related to NewtonWigner localization (for the details see $[13,14]$ ). Using the explicit form of gamma matrices (74), one can show that $\hat{\mathbf{S}}_{N W}$ is equal to the spin operator given in Eq. (42). Therefore, we can write

$$
\langle\mathbf{S}\rangle=\operatorname{Tr}\left(\hat{\mathbf{S}}_{N W} \Omega(k)\right) .
$$

\section{Unitary, covariant evolution of a qubit}

Formalism introduced in the previous section enables us to find a model of Lorentzcovariant and unitary evolution of a Majorana qubit. To this end, let us consider the following element of the bispinor representation of the $\operatorname{SL}(2, \mathbb{C})$ group:

$$
K(\tau)=e^{-i \tau F}=e^{-i \tau(\boldsymbol{\omega} \cdot \mathbf{J}+\eta \cdot \mathbf{K})},
$$

where the most general form of $F$ reads

$$
F=f_{\mu \nu} \frac{i}{4}\left[\gamma^{\mu}, \gamma^{\nu}\right]=f_{i j} \frac{i}{4}\left[\gamma^{i}, \gamma^{j}\right]+f_{0 j} \frac{i}{4}\left[\gamma^{0}, \gamma^{j}\right] \equiv \boldsymbol{\omega} \cdot \mathbf{J}+\boldsymbol{\eta} \cdot \mathbf{K} .
$$

In the above equation, we have introduced the standard notation for generators of rotations and pure Lorentz boosts

$$
J^{k}=\frac{i}{8} \varepsilon_{k i j}\left[\gamma^{i}, \gamma^{j}\right], \quad K^{j}=\frac{i}{4}\left[\gamma^{0}, \gamma^{j}\right]
$$


The explicit form of $\mathbf{J}$ and $\mathbf{K}$ in the Weyl representation of gamma matrices used in this paper is the following:

$$
\mathbf{J}=\frac{1}{2}\left(\begin{array}{ll}
\sigma & 0 \\
0 & \sigma
\end{array}\right), \quad \mathbf{K}=\frac{i}{2}\left(\begin{array}{cc}
\sigma & 0 \\
0 & -\sigma
\end{array}\right)
$$

Now, we identify $\tau$ in $K(\tau)$ (Eq. (47)) with a proper time of the particle. Transformations $K(\tau)$ form one-parameter subgroup of a bispinor representation of the $\mathrm{SL}(2, \mathbb{C})$ group. The whole family $\{K(\tau)\}$ transforms in an explicitly covariant manner under inner automorphisms of the $\operatorname{SL}(2, \mathbb{C})$ group. Thus, equivalent trajectories are determined by the values of invariants corresponding to Casimir operators in the Lie algebra of the $\mathrm{SL}(2, \mathbb{C})$ group. These invariants read:

$$
C_{1}=\omega^{2}-\eta^{2}=\omega^{2}-\eta^{2}, \quad C_{2}=\omega \cdot \eta=\omega \eta \cos \theta .
$$

In the bispinor representation, $\gamma^{\mu}$ transforms like four-vector; therefore,

$$
K(\tau) q_{\mu} \gamma^{\mu} \bar{K}(\tau)=p_{\mu}(\tau) \gamma^{\mu}, \quad q_{\mu}=p_{\mu}(0) .
$$

In the following, $p_{\mu}(\tau)$ will be identified with a four-momentum of an evolving Majorana qubit.

Taking into account the form of $K(\tau)$ and Eq. (52), one can easily show that the Minkowski square of a four-acceleration

$$
a^{\mu}(\tau)=\frac{1}{m} \frac{d p^{\mu}(\tau)}{d \tau}
$$

is negative and independent of a proper time $\tau$. This means that a motion defined by (52) is an uniformly accelerated motion. Furthermore, when we determine $p^{\mu}(\tau)$ we can estimate a classical trajectory of a qubit using the formula

$$
\mathbf{x}(\tau)=\frac{1}{m} \int_{0}^{\tau} \mathbf{p}\left(\tau^{\prime}\right) d \tau^{\prime}
$$

Now, choosing various $C_{1}$ and $C_{2}$ we obtain different types of motion. In particular, we can distinguish the following special types:

1. $\omega=\eta \neq 0, \omega \perp \eta\left(C_{1}=C_{2}=0\right)$

2. $\omega=0\left(C_{2}=0, C_{1}<0\right)$

3. $\eta=0\left(C_{2}=0, C_{1}>0\right)$

Of course, in general motions with arbitrary values of $C_{1}$ and $C_{2}$ are possible. Analogous classification of uniformly accelerated motions was considered in $[17,18,20]$.

Next, by means of Weinberg conditions (8) we can connect the action of oneparametric subgroup $K(\tau) \in \mathrm{SL}(2, \mathbb{C})$ in bispinor basis with its action on objects in spin basis (and vice versa). Formulas $(27,28)$ and (8) imply that inserting $A=K(\tau)$ we get

$$
\Omega^{\prime}(p(\tau), \boldsymbol{\xi}(\tau, q))=K(\tau) \Omega(q, \boldsymbol{\xi}(0, q)) K^{-1}(\tau),
$$


and

$$
\rho(\boldsymbol{\xi}(\tau, q))=\mathbb{W}(K(\tau), q) \rho(\boldsymbol{\xi}(0, q)) \mathbb{W}^{\dagger}(K(\tau), q),
$$

where the unitary matrix $\mathbb{W}(K(\tau), q)$ represents the Wigner rotation $L_{K(\tau) q}^{-1} K(\tau) L_{q}$ compare Eq. (79). In this way explicitly covariant (but in general nonunitary) transformation of a matrix $\Omega$ is realized as unitary transformation of a corresponding density matrix $\rho$.

Using the form of $\Omega$ given in Eq. (35), we obtain

$$
\Omega(p(\tau), w(\tau))=K(\tau) \Omega(q, w(0)) K^{-1}(\tau),
$$

where $w(0)$ can be determined from Eq. (33) by inserting $q^{\mu}$ as a four-momentum and $\xi(0)$ as a polarization vector.

$w^{\mu}(\tau)$ is a (pseudo-)four-vector; therefore, excluding space-time inversions, it transforms like $p^{\mu}(\tau)$. Using this observation, we can determine $w^{\mu}(\tau)$ with the help of the following formula:

$$
w_{\mu}(\tau) \gamma^{\mu}=K(\tau) w_{\mu}(0) \gamma^{\mu} \bar{K}(\tau)
$$

Having $w(\tau)$ and $p(\tau)$ one can calculate $\xi(\tau, q)$ from Eq. (33). Alternatively, we can determine a Wigner rotation $\mathbb{W}(K(\tau), q)$ and eventually $\rho(\boldsymbol{\xi}(\tau, q))$.

In general formulas for $p^{\mu}(\tau)$ and $\boldsymbol{\xi}(\tau, q)$ for arbitrary $q^{\mu}, \boldsymbol{\xi}(0, q), \omega$ and $\eta$ obtained from (52) and (58) are very long. Therefore, we will not present them here explicitly, but instead we illustrate our considerations by characteristic examples.

First, let us consider the simplest case $\omega=\eta \neq 0, \omega \perp \eta\left(C_{1}=C_{2}=0\right)$. Using this case, we will illustrate all steps that are necessary to find formulas for $p^{\mu}(\tau)$ and $\boldsymbol{\xi}(\tau, q)$. We start with four-momentum. With the help of (52), we find explicitly:

$$
\begin{aligned}
p^{0}(\tau) & =2 \tau^{2}\left[q^{0} \omega^{2}+\mathbf{q} \cdot(\boldsymbol{\eta} \times \boldsymbol{\omega})\right]+2 \tau(\mathbf{q} \cdot \boldsymbol{\eta})+q^{0}, \\
\mathbf{p}(\tau) & =2 \tau^{2}\left[(\mathbf{q} \cdot \boldsymbol{\eta}) \boldsymbol{\eta}+(\mathbf{q} \cdot \boldsymbol{\omega}) \boldsymbol{\omega}-q^{0}(\boldsymbol{\eta} \times \boldsymbol{\omega})-\omega^{2} \mathbf{q}\right]+2 \tau\left[q^{0} \boldsymbol{\eta}-(\mathbf{q} \times \boldsymbol{\omega})\right]+\mathbf{q} .
\end{aligned}
$$

The trajectory can be determined from (54). We receive

$$
\begin{aligned}
x^{0}(\tau) & =\frac{2 \tau^{3}}{3 m}\left[q^{0} \omega^{2}+\mathbf{q} \cdot(\boldsymbol{\eta} \times \boldsymbol{\omega})\right]+\frac{\tau^{2}}{m}(\mathbf{q} \cdot \boldsymbol{\eta})+\frac{\tau}{m} q^{0} \\
\mathbf{x}(\tau) & =\frac{2 \tau^{3}}{3 m}\left[(\mathbf{q} \cdot \boldsymbol{\eta}) \boldsymbol{\eta}+(\mathbf{q} \cdot \boldsymbol{\omega}) \boldsymbol{\omega}-q^{0}(\boldsymbol{\eta} \times \boldsymbol{\omega})-\omega^{2} \mathbf{q}\right]+\frac{\tau^{2}}{m}\left[q^{0} \boldsymbol{\eta}-(\mathbf{q} \times \boldsymbol{\omega})\right]+\frac{\tau}{m} \mathbf{q} .
\end{aligned}
$$

Let us notice that we can interpret Eq. (61) as a relationship between a coordinate time $x^{0}$ and a proper time $\tau$. We can invert this relation to obtain $\tau$ as a function of coordinate time.

As a next step, we determine a four-acceleration using (53). We get

$$
a^{0}(\tau)=\frac{4}{m} \tau\left[q^{0} \omega^{2}+\mathbf{q} \cdot(\boldsymbol{\eta} \times \boldsymbol{\omega})\right]+\frac{2}{m}(\mathbf{q} \cdot \boldsymbol{\eta}),
$$




$$
\mathbf{a}(\tau)=\frac{4}{m} \tau\left[(\mathbf{q} \cdot \boldsymbol{\eta}) \boldsymbol{\eta}+(\mathbf{q} \cdot \boldsymbol{\omega}) \boldsymbol{\omega}-q^{0}(\boldsymbol{\eta} \times \boldsymbol{\omega})-\omega^{2} \mathbf{q}\right]+\frac{2}{m}\left[q^{0} \boldsymbol{\eta}-(\mathbf{q} \times \boldsymbol{\omega})\right],
$$

and

$$
a^{2}=a^{\mu}(\tau) a_{\mu}(\tau)=\frac{4}{m^{2}}\left[\omega^{2} m^{2}+(\mathbf{q} \cdot \boldsymbol{\eta})^{2}+(\mathbf{q} \cdot \boldsymbol{\omega})^{2}-2 q^{0}\left[q^{0} \omega^{2}+\mathbf{q} \cdot(\boldsymbol{\eta} \times \boldsymbol{\omega})\right]\right] .
$$

We want to obtain the evolution of a polarization vector $\boldsymbol{\xi}(\tau)$. To this end, we first determine a Pauli-Lubanski four-vector as a function of $\tau$. Using (58), we obtain

$$
\begin{aligned}
w^{0}(\tau)= & \tau^{2}\left[\omega^{2}(\mathbf{q} \cdot \zeta)+m \zeta \cdot(\boldsymbol{\eta} \times \boldsymbol{\omega})+\kappa \mathbf{q} \cdot(\boldsymbol{\eta} \times \boldsymbol{\omega})\right] \\
+ & \tau[m(\boldsymbol{\eta} \cdot \boldsymbol{\zeta})+\kappa(\mathbf{q} \cdot \boldsymbol{\eta})]+\frac{\mathbf{q} \cdot \boldsymbol{\zeta}}{2} \\
\mathbf{w}(\tau)= & \tau^{2}\left[\kappa\left[(\mathbf{q} \cdot \boldsymbol{\eta}) \boldsymbol{\eta}+(\mathbf{q} \cdot \boldsymbol{\omega}) \boldsymbol{\omega}-\omega^{2} \mathbf{q}\right]+m\left[(\boldsymbol{\eta} \cdot \boldsymbol{\zeta}) \boldsymbol{\eta}+(\boldsymbol{\omega} \cdot \zeta) \boldsymbol{\omega}-\omega^{2} \zeta\right]\right. \\
& \quad-(\mathbf{q} \cdot \boldsymbol{\zeta})(\boldsymbol{\eta} \times \boldsymbol{\omega})] \\
+ & \tau[(\mathbf{q} \cdot \zeta) \boldsymbol{\eta}+m(\boldsymbol{\omega} \times \zeta)-\kappa(\mathbf{q} \times \boldsymbol{\omega})]+\frac{1}{2}(m \zeta+\kappa \mathbf{q})
\end{aligned}
$$

where for notational simplicity we have denoted $\zeta=\xi(0, q)$ and $\kappa=\frac{\mathbf{q} \cdot \boldsymbol{\zeta}}{m+q^{0}}$.

Finally, $\boldsymbol{\xi}(\tau, q)$ can be obtained from (33) via the relation

$$
\boldsymbol{\xi}(\tau)=\frac{2}{m}\left(\mathbf{w}(\tau)-\frac{w^{0}(\tau)}{m+p^{0}(\tau)} \mathbf{p}(\tau)\right)
$$

After tedious calculation, we have

$$
\boldsymbol{\xi}(\tau, q)=\zeta+\frac{2 \tau A+2 \tau^{2} B+4 \tau^{3} C+4 \tau^{4} D}{m+q^{0}+2 \tau(\mathbf{q} \cdot \boldsymbol{\eta})+2 \tau^{2}\left[q^{0} \omega^{2}+\mathbf{q} \cdot(\boldsymbol{\eta} \times \boldsymbol{\omega})\right]},
$$

where

$$
\begin{gathered}
A=(\mathbf{q} \cdot \zeta) \boldsymbol{\eta}-(\boldsymbol{\eta} \cdot \boldsymbol{\zeta}) \mathbf{q}+\left(m+q^{0}\right)(\boldsymbol{\omega} \times \boldsymbol{\zeta}), \\
B=\kappa\left[2(\mathbf{q} \cdot \boldsymbol{\eta}) \boldsymbol{\eta}-\omega^{2} \mathbf{q}\right]-\left(m+q^{0}\right) \omega^{2} \boldsymbol{\zeta}+\left(m+q^{0}\right)(\boldsymbol{\omega} \cdot \boldsymbol{\zeta}) \boldsymbol{\omega}+\left(m-q^{0}\right)(\boldsymbol{\eta} \cdot \boldsymbol{\zeta}) \boldsymbol{\eta} \\
-\boldsymbol{\zeta} \cdot(\boldsymbol{\eta} \times \boldsymbol{\omega}) \mathbf{q}+2(\boldsymbol{\eta} \cdot \boldsymbol{\zeta})(\mathbf{q} \times \boldsymbol{\omega})+2(\mathbf{q} \cdot \boldsymbol{\eta})(\boldsymbol{\omega} \times \boldsymbol{\zeta})-(\mathbf{q} \cdot \boldsymbol{\zeta})(\boldsymbol{\eta} \times \boldsymbol{\omega}), \\
C=\kappa\left[\mathbf{q} \cdot(\boldsymbol{\eta} \times \boldsymbol{\omega}) \boldsymbol{\eta}+\omega^{2}(\mathbf{q} \times \boldsymbol{\omega})-(\mathbf{q} \cdot \boldsymbol{\eta})(\boldsymbol{\eta} \times \boldsymbol{\omega})\right]+(\boldsymbol{\zeta} \cdot(\boldsymbol{\eta} \times \boldsymbol{\omega}))(\mathbf{q} \times \boldsymbol{\omega}) \\
+[(\mathbf{q} \cdot \boldsymbol{\eta})(\boldsymbol{\omega} \cdot \boldsymbol{\zeta})-(\mathbf{q} \cdot \boldsymbol{\omega})(\boldsymbol{\eta} \cdot \boldsymbol{\zeta})] \boldsymbol{\omega}+\left[q^{0} \omega^{2}+\mathbf{q} \cdot(\boldsymbol{\eta} \times \boldsymbol{\omega})\right](\boldsymbol{\omega} \times \boldsymbol{\zeta}) \\
-q^{0}(\boldsymbol{\zeta} \cdot(\boldsymbol{\eta} \times \boldsymbol{\omega})) \boldsymbol{\eta}+q^{0}(\boldsymbol{\eta} \cdot \boldsymbol{\zeta})(\boldsymbol{\eta} \times \boldsymbol{\omega})+\omega^{2}(\boldsymbol{\eta} \cdot \boldsymbol{\zeta}) \mathbf{q}-\omega^{2}(\mathbf{q} \cdot \boldsymbol{\eta}) \boldsymbol{\zeta},
\end{gathered}
$$




$$
\begin{aligned}
D & =\kappa\left[\omega^{4} \mathbf{q}-\omega^{2}(\mathbf{q} \cdot \boldsymbol{\eta}) \boldsymbol{\eta}-\omega^{2}(\mathbf{q} \cdot \boldsymbol{\omega}) \boldsymbol{\omega}-(\mathbf{q} \cdot(\boldsymbol{\eta} \times \boldsymbol{\omega}))(\boldsymbol{\eta} \times \boldsymbol{\omega})\right] \\
& +\left[q^{0} \omega^{2}(\boldsymbol{\omega} \cdot \boldsymbol{\zeta})+(\boldsymbol{\omega} \cdot \boldsymbol{\zeta})(\mathbf{q} \cdot(\boldsymbol{\eta} \times \boldsymbol{\omega}))-(\mathbf{q} \cdot \boldsymbol{\omega})(\boldsymbol{\zeta} \cdot(\boldsymbol{\eta} \times \boldsymbol{\omega}))\right] \boldsymbol{\omega} \\
& +\left[q^{0} \omega^{2}(\boldsymbol{\eta} \cdot \boldsymbol{\zeta})+(\boldsymbol{\eta} \cdot \boldsymbol{\zeta})(\mathbf{q} \cdot(\boldsymbol{\eta} \times \boldsymbol{\omega}))-(\mathbf{q} \cdot \boldsymbol{\eta})(\boldsymbol{\zeta} \cdot(\boldsymbol{\eta} \times \boldsymbol{\omega}))\right] \boldsymbol{\eta} \\
& +q^{0}(\boldsymbol{\zeta} \cdot(\boldsymbol{\eta} \times \boldsymbol{\omega}))(\boldsymbol{\eta} \times \boldsymbol{\omega})-\omega^{2}\left[q^{0} \omega^{2}+\mathbf{q} \cdot(\boldsymbol{\eta} \times \boldsymbol{\omega})\right] \zeta+\omega^{2}(\zeta \cdot(\boldsymbol{\eta} \times \boldsymbol{\omega})) \mathbf{q}
\end{aligned}
$$

In Fig. 1, we have depicted $\mathbf{p}(\tau), \mathbf{x}(\tau)$ and $\boldsymbol{\xi}(\tau)$ for chosen initial conditions. For plots we assume, without loss of generality, that $m=1$ and the initial momentum is directed along $z$-axis, $q^{\mu}=\left(\sqrt{1+q^{2}}, 0,0, q\right)$. Again, without loss of generality we take $\omega$ in $x-z$ plane, $\omega=\omega(\cos \alpha, 0, \sin \alpha)$ and $\eta$ as an arbitrary vector such that $|\eta|=|\omega|, \eta \perp \omega$. As an initial polarization vector we take an arbitrary unit vector, $\xi=(\cos \phi \sin \Omega, \sin \phi \sin \Omega, \cos \Omega)$. In Fig. 1a we present the evolution of momentum $\mathbf{p}(\tau)$, in Fig. 1b a classical trajectory of a qubit $\mathbf{x}(\tau)$ and in Fig. 1c an evolution of a polarization vector $\xi(\tau, q)$ for $\tau \in\left\langle 0, \tau_{\max }\right\rangle$. In all three plots, we use the same initial conditions. Points in plots $1 \mathrm{a}, \mathrm{b}$ correspond to proper times: 0 , $\frac{1}{3} \tau_{\max }, \frac{2}{3} \tau_{\max }$. In plot $1 \mathrm{c}$ we have depicted the trajectory of a polarization vector $\xi$ on a sphere $|\xi|=$ const (notice that unitary evolution (56) preserves the length of a polarization vector, $|\boldsymbol{\xi}(\tau, q)|=$ const $)$. Arrows correspond to $\boldsymbol{\xi}(0), \boldsymbol{\xi}\left(\frac{1}{3} \tau_{\max }\right), \boldsymbol{\xi}\left(\frac{2}{3} \tau_{\max }\right)$, and to the limit $\xi(\tau \rightarrow \infty)$. Notice that the end of the polarization vector describes a fragment of a circle on the Bloch sphere. It is a general feature of the considered case $|\omega|=|\eta| \neq 0, \omega \perp \eta$.

As we have seen, general formulas for $x^{\mu}(\tau), p^{\mu}(\tau), w^{\mu}(\tau), \xi(\tau)$ are rather long even in the simplest case $|\boldsymbol{\omega}|=|\boldsymbol{\eta}| \neq 0, \boldsymbol{\omega} \perp \boldsymbol{\eta}$. In all other cases, the corresponding formulas are much longer. Therefore, we do not present them here explicitly but rather describe general features of each of the cases.

In the case of the hyperbolic motion $(\boldsymbol{\omega}=\mathbf{0}, \boldsymbol{\eta} \neq \mathbf{0})$, the trajectory of a particle is situated in a plane determined by the initial momentum $\mathbf{q}$ and the vector $\boldsymbol{\eta}$. Let us stress that in this case it is possible to arrange initial conditions in such a way that the quantum spin state does not change during the evolution. It holds when the initial momentum $\mathbf{q}$ is parallel to the vector $\eta$.

In the case of the circular motion $(\boldsymbol{\omega} \neq \mathbf{0}, \boldsymbol{\eta}=\mathbf{0})$, the trajectory in general is a helix, except the special case $\mathbf{q} \perp \boldsymbol{\omega}$ when the trajectory is a circle. The end of the Bloch vector moves on a circle on a Bloch sphere. Notice that when the initial momentum $\mathbf{q}=\mathbf{0}$ the particle stays at rest but the polarization vector rotates.

The most complicated behaviour we have in a general situation $C_{1} \neq 0, C_{2} \neq 0$. Therefore, as a next example we consider the case $\omega \| \boldsymbol{\eta}$ belonging to this class. In Figs. 2 and 3, we have depicted $\mathbf{p}(\tau), \mathbf{x}(\tau)$ and $\boldsymbol{\xi}(\tau)$ for chosen initial conditions. For plots, we assume as in the previous case that $m=1$ and the initial momentum is directed along $z$-axis, $q^{\mu}=\left(\sqrt{1+q^{2}}, 0,0, q\right)$. Again, without loss of generality we take $\omega$ in $x-z$ plane, $\omega=\omega(\cos \alpha, 0, \sin \alpha)$. Now, for simplicity we assume that $\eta$ is parallel to $\omega$ but has different magnitude, i.e. $\eta=\eta(\cos \alpha, 0, \sin \alpha)$. As an initial polarization vector, we take an arbitrary unit vector, $\xi=(\cos \phi \sin \Omega, \sin \phi \sin \Omega, \cos \Omega)$. In Figs. 2a and $3 \mathrm{a}$ we have depicted the evolution of momentum $\mathbf{p}(\tau)$, in Figs. $2 \mathrm{~b}$ and $3 \mathrm{~b}$ classical trajectory of a qubit $\mathbf{x}(\tau)$ and in Figs. $2 \mathrm{c}$ and $3 \mathrm{c}$ evolution of a polarization vector $\boldsymbol{\xi}(\tau, q)$ for $\tau \in\left\langle 0, \tau_{\max }\right\rangle$. Like in Fig. 1, in all three plots we use the same initial 
(a)

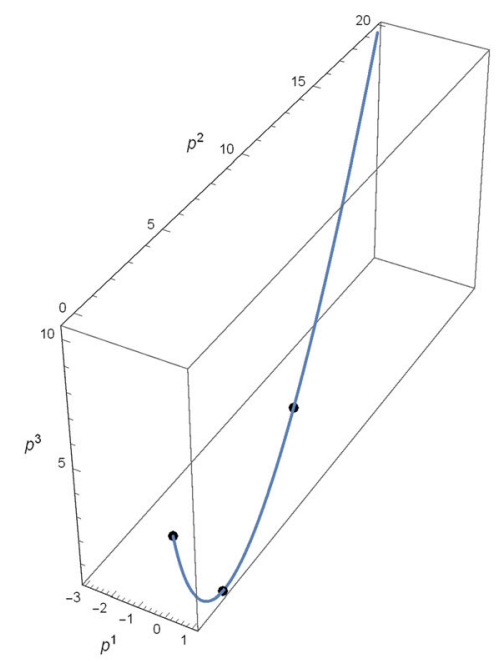

(b)

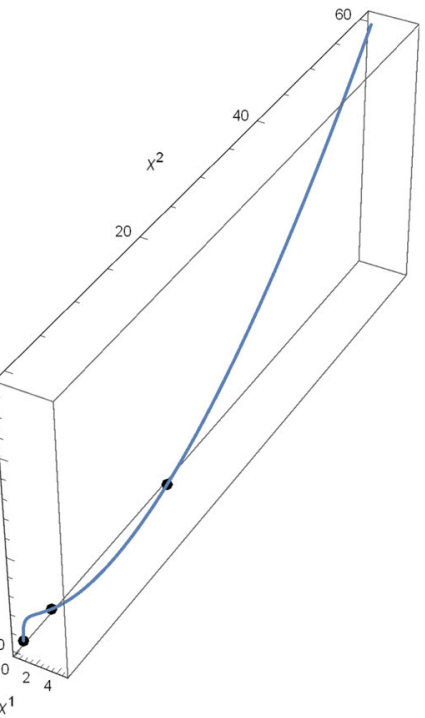

(c)

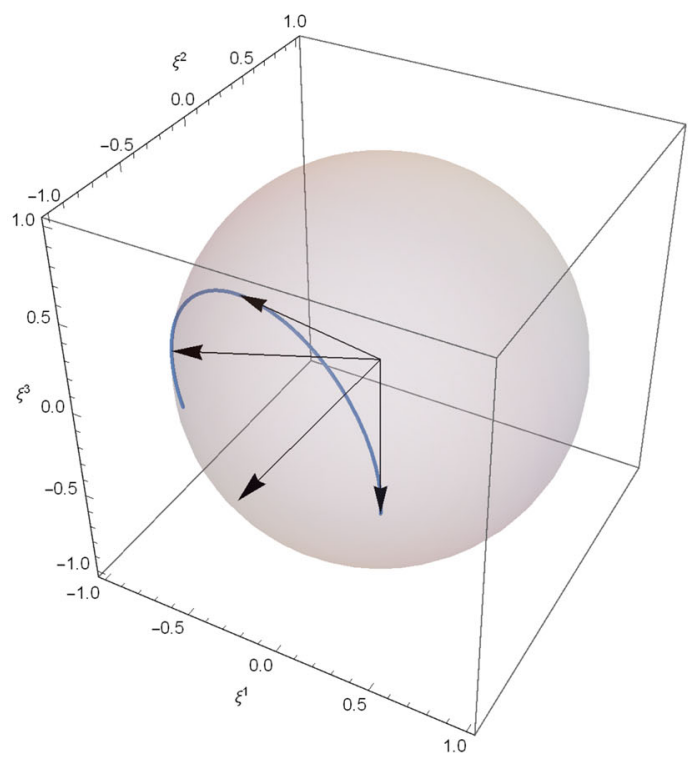

Fig. 1 Evolution in the case $\omega=\eta \neq 0, \omega \perp \eta$. We assume that $m=1,|\xi|=1$ and $q^{\mu}=\left(\sqrt{1+q^{2}}, 0,0, q\right), \omega=\omega(\cos \alpha, 0, \sin \alpha), \eta=\omega(\sin \alpha \sin \beta, \cos \beta,-\cos \alpha \sin \beta), \xi(0, q)=$ $(\cos \phi \sin \Omega, \sin \phi \sin \Omega, \cos \Omega$ ). In a we present the evolution of the momentum $\mathbf{p}(\tau)$, in $\mathbf{b}$ we present the classical trajectory $\mathbf{x}(\tau)$ and in $\mathbf{c}$ we present the evolution of the polarization vector $\boldsymbol{\xi}(\tau)$. All plots are drawn for the following initial conditions: $q=3, \omega=0.4, \alpha=\pi / 8, \beta=\pi / 4, \phi=\pi / 8, \Omega=\pi$. All of the plots are drawn for the proper time $\tau \in\left\langle 0, \tau_{\max }=9.6\right\rangle$. Points and arrows correspond to $\tau=0$, $\tau=\tau_{\max } / 3, \tau=2 \tau_{\max } / 3$. The fourth arrow in the plot (c) corresponds to the limit $\xi(\tau \rightarrow \infty)$ 
(a)

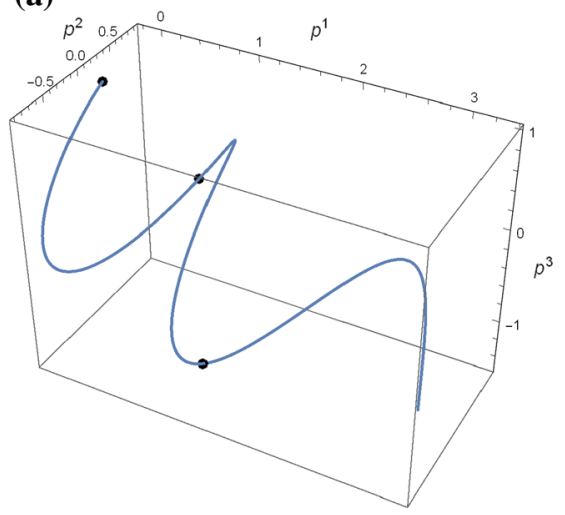

(b)

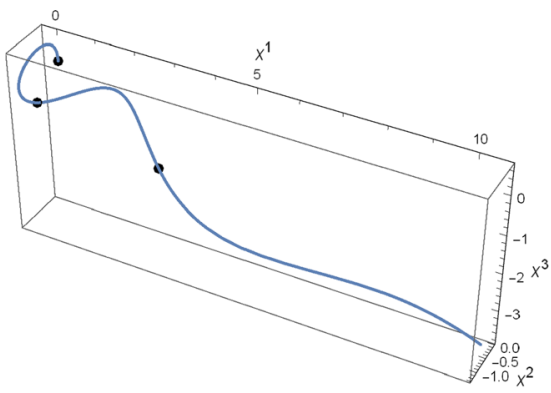

(c)

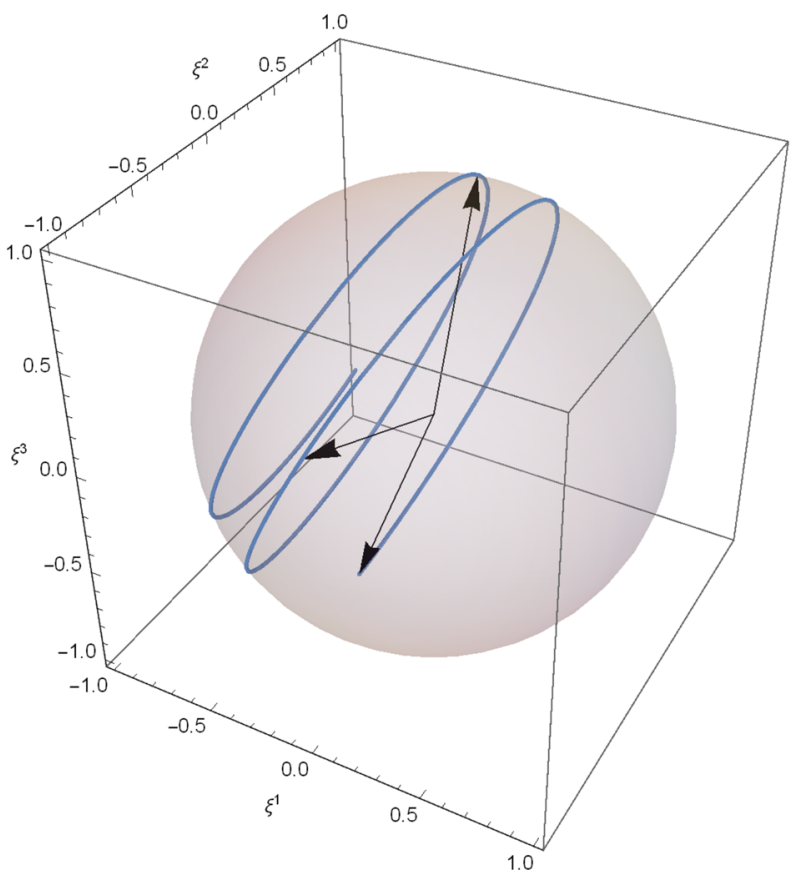

Fig. 2 Evolution in the case $\omega|| \eta$. We assume that $m=1,|\xi|=1$ and $q^{\mu}=\left(\sqrt{1+q^{2}}, 0,0, q\right), \omega=$ $\omega(\cos \alpha, 0, \sin \alpha), \eta=\eta(\cos \alpha, 0, \sin \alpha), \xi(0, q)=(\cos \phi \sin \Omega, \sin \phi \sin \Omega, \cos \Omega)$. In a we present the evolution of the momentum $\mathbf{p}(\tau)$, in $\mathbf{b}$ we present the classical trajectory $\mathbf{x}(\tau)$ and in $\mathbf{c}$ we present the evolution of the polarization vector $\boldsymbol{\xi}(\tau)$. All plots are drawn for the following initial conditions: $q=1$, $\omega=0.7, \eta=0.1 \alpha=3 \pi / 8, \phi=\pi / 10, \Omega=10 \pi / 9$. All of the plots are drawn for the proper time $\tau \in\left\langle 0, \tau_{\max }=9.6\right\rangle$. Points and arrows correspond to $\tau=0, \tau=\tau_{\max } / 3, \tau=2 \tau_{\max } / 3$ 
(a)

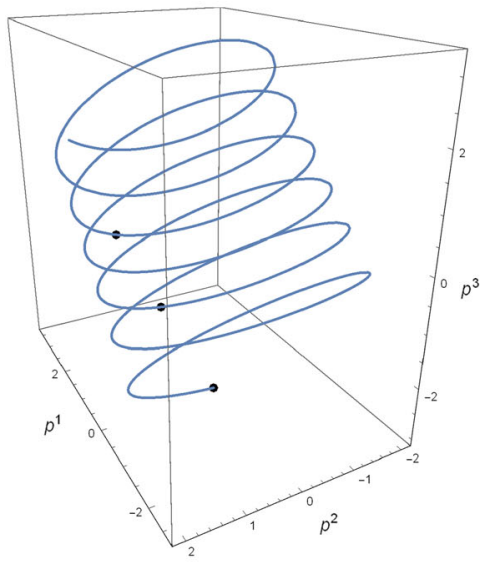

(b)

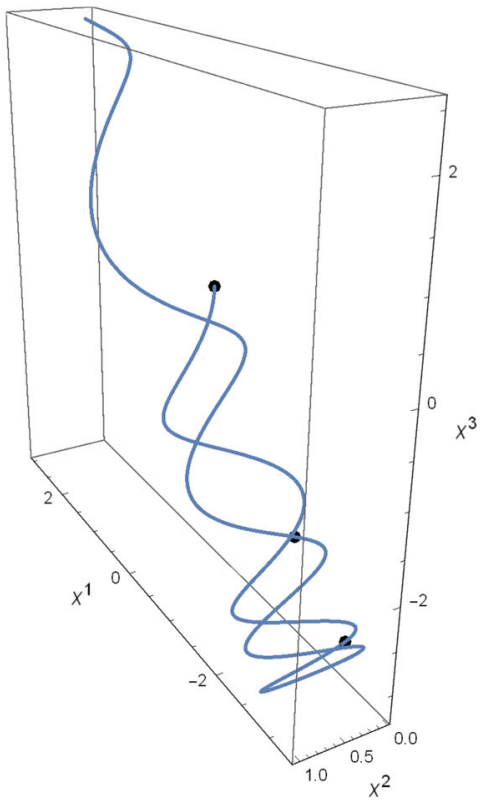

(c)

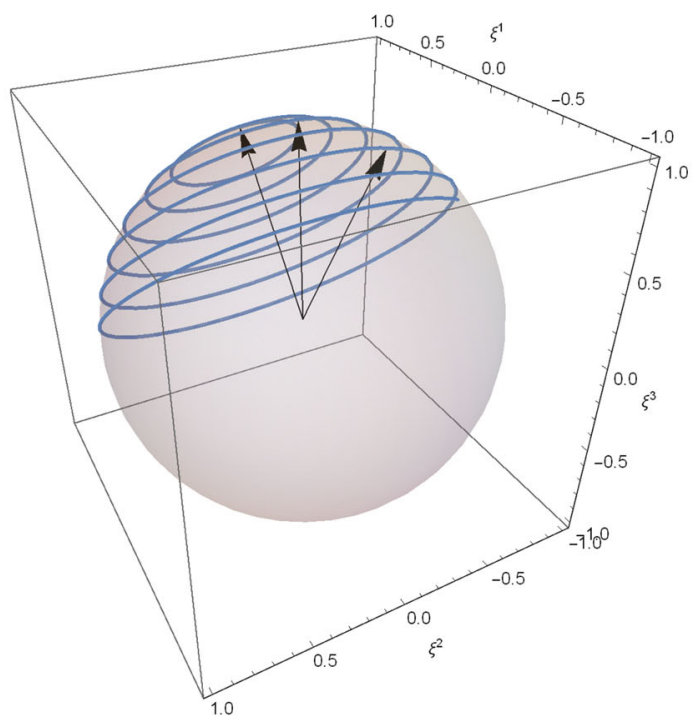

Fig. 3 Evolution in the case $\omega \| \boldsymbol{\eta}$. As in Fig. 2, we assume that $m=1,|\boldsymbol{\xi}|=1$ and $q^{\mu}=\left(\sqrt{1+q^{2}}, 0,0, q\right)$, $\omega=\omega(\cos \alpha, 0, \sin \alpha), \eta=\eta(\cos \alpha, 0, \sin \alpha), \xi(0, q)=(\cos \phi \sin \Omega, \sin \phi \sin \Omega, \cos \Omega)$. In Fig. 2a we present the evolution of the momentum $\mathbf{p}(\tau)$, in Fig. $2 \mathrm{~b}$ we present the classical trajectory $\mathbf{x}(\tau)$ and in Fig. $2 \mathrm{c}$ we present the evolution of the polarization vector $\boldsymbol{\xi}(\tau)$. All plots are drawn for the following initial conditions: $q=-3, \omega=2, \eta=0.1 \alpha=\pi / 4, \phi=\pi / 18, \Omega=\pi / 6$. All of the plots are drawn for the proper time $\tau \in\left\langle 0, \tau_{\max }=9.6\right\rangle$. Points and arrows correspond to $\tau=0, \tau=\tau_{\max } / 3, \tau=2 \tau_{\max } / 3$ 
conditions. Points in plots $2 \mathrm{a}, \mathrm{b} 3 \mathrm{a}, \mathrm{b}$ correspond to proper times: $0, \frac{1}{3} \tau_{\max }, \frac{2}{3} \tau_{\max }$. In plots $2 \mathrm{c}$ and $3 \mathrm{c}$, we have depicted the trajectory of a polarization vector $\xi$ on a sphere $|\xi|=$ const (as in the previous example, unitary evolution (56) preserves the length of a polarization vector, $|\boldsymbol{\xi}(\tau, q)|=$ const $)$. Arrows correspond to $\boldsymbol{\xi}(0), \boldsymbol{\xi}\left(\frac{1}{3} \tau_{\max }\right), \boldsymbol{\xi}\left(\frac{2}{3} \tau_{\max }\right)$. We see that in this case the end of the polarization vector describes quite a complicated curve on the Bloch sphere. The trajectory of the Majorana particle is a composition of a circular and hyperbolic motion; its detailed shape depends on initial condition.

\section{Conclusions}

We have defined a relativistic spin density matrix for a Majorana qubit, using ideas developed in [11] where a covariant description is formulated within the four-dimensional (bispinor) formalism. We have also discussed an appropriate representation of a spin observable in a covariant basis.

Next, we have developed a model of a time evolution of the density matrix. This evolution is manifestly Lorentz covariant in the bispinor basis while unitary in the spin basis. Treating four-momentum as an external classical parameter, we can determine the classical trajectory of a particle. Such an approach allows us to treat a Majorana particle classically with respect to the evolution of the kinematical parameters, while quantum with respect to the evolution of the spin. We have shown that in our model the motion on a classical trajectory is an uniformly accelerated motion (the square of a fouracceleration is constant), we have also distinguished different types of these motions (see also $[17,18,20])$. In the simplest case, we have derived explicit formulas for $p^{\mu}(\tau), a^{\mu}(\tau), x^{\mu}(\tau), w^{\mu}(\tau)$, and $\xi(\tau)$. The obtained formulas are quite complicated even in this case. Therefore, the second, more general, example we have treated only numerically. We have depicted the behaviour of $\mathbf{p}(\tau), \mathbf{x}(\tau)$, and $\boldsymbol{\xi}(\tau)$ in a number of plots. As an evolution parameter, we have taken the proper time $\tau$, but one can instead use a coordinate time-compare the discussion after (62).

We hope that the proposed model of evolution will be useful in the discussion of correlation experiments with accelerated particles.

Funding Funding was provided by Narodowe Centrum Nauki (Grant No. 2014/15/B/ST2/00117), and by University of Lodz.

Open Access This article is distributed under the terms of the Creative Commons Attribution 4.0 International License (http://creativecommons.org/licenses/by/4.0/), which permits unrestricted use, distribution, and reproduction in any medium, provided you give appropriate credit to the original author(s) and the source, provide a link to the Creative Commons license, and indicate if changes were made.

\section{A Conventions}

Dirac matrices fulfil the relation $\gamma^{\mu} \gamma^{\nu}+\gamma^{\nu} \gamma^{\mu}=2 g^{\mu \nu}$, where we choose the Minkowski metric tensor $g^{\mu \nu}=\operatorname{diag}(1,-1,-1,-1)$; we also take $\varepsilon^{0123}=1$. The 
following explicit representation of gamma matrices is used throughout the paper:

$$
\gamma^{0}=\left(\begin{array}{ll}
0 & I \\
I & 0
\end{array}\right), \quad \gamma=\left(\begin{array}{cc}
0 & -\sigma \\
\sigma & 0
\end{array}\right), \quad \gamma^{5}=\left(\begin{array}{cc}
I & 0 \\
0 & -I
\end{array}\right)
$$

where $\sigma=\left(\sigma_{1}, \sigma_{2}, \sigma_{3}\right)$ and $\sigma_{i}$ are standard Pauli matrices.

The charge conjugation matrix has the form

$$
\mathscr{C}=-i \gamma^{2} \gamma^{0}=i\left(\begin{array}{cc}
\sigma_{2} & 0 \\
0 & -\sigma_{2}
\end{array}\right)
$$

so

$$
\mathscr{C} \gamma^{\mu T} \mathscr{C}^{-1}=-\gamma^{\mu}
$$

and

$$
\mathscr{C} \mathscr{C}^{\dagger}=I
$$

The bispinor representation of the Lorentz group is the representation $D^{\left(\frac{1}{2}, 0\right)} \oplus$ $D^{\left(0, \frac{1}{2}\right)}$. Explicitly, if $A \in \mathrm{SL}(2, \mathbb{C})$ and $\Lambda(A)$ is an image of $A$ in the canonical homomorphism of the $\mathrm{SL}(2, \mathbb{C})$ group onto the Lorentz group, we take

$$
S(\Lambda(A))=\left(\begin{array}{cc}
A & 0 \\
0 & \left(A^{\dagger}\right)^{-1}
\end{array}\right)
$$

(so-called Weyl or chiral form of the bispinor representation). The canonical homomorphism between the group $\operatorname{SL}(2, \mathbb{C})$ (universal covering of the proper orthochronous Lorentz group $\left.L_{+}^{\uparrow}\right)$ and the Lorentz group $L_{+}^{\uparrow} \sim \mathrm{SO}(1,3)_{0}$ is defined as follows [3]: With every four-vector $k^{\mu}$ we associate a two-dimensional Hermitian matrix $k^{\mu} \sigma_{\mu}$, where $\sigma_{0}=I$ and $\sigma_{i}, i=1,2,3$, are the standard Pauli matrices. In the space of two-dimensional Hermitian matrices, the Lorentz group action is given by $k^{\prime \mu} \sigma_{\mu}=A\left(\mathrm{k}^{\mu} \sigma_{\mu}\right) A^{\dagger}$, where $A$ denotes the element of the $\operatorname{SL}(2, \mathbb{C})$ group corresponding to the Lorentz transformation $\Lambda(A)$ which converts the four-vector $k$ to $k^{\prime}$ (i.e. $\left.k^{\prime \mu}=\Lambda^{\mu}{ }_{\nu} k^{v}\right)$.

In our paper, we use blackboard bold typeface style to denote matrices from $\operatorname{SL}(2, \mathbb{C})$ group corresponding to the Lorentz transformations. In particular, we use the following transformations

- $\mathbb{W}(\Lambda, k)$ corresponding to the Wigner rotation

$$
R(\Lambda, k)=L_{\Lambda k}^{-1} \Lambda L_{k}
$$

where $L_{k}$ denotes the standard Lorentz boost defined by the relations $L_{k} \tilde{k}=$ $k, L_{\tilde{k}}=I, \tilde{k}=(m, \mathbf{0})$. In fact, $\mathbb{W}(\Lambda, k)$ coincides with the matrix spin $1 / 2$ representation of the $R(\Lambda, k) \in \mathrm{SO}(3), \mathbb{W}(\Lambda, k)=\mathscr{D}^{1 / 2}(R(\Lambda, k))$. 
- $\mathbb{L}_{k}$ corresponding to the standard Lorentz boost defined by the relations $L_{k} \tilde{k}=k$, $L_{\tilde{k}}=I, \tilde{k}=(m, \mathbf{0})$. Explicitly

$$
\mathbb{L}_{k}=\frac{1}{\sqrt{2\left(1+\frac{k^{0}}{m}\right)}}\left(I+\frac{k^{\mu} \sigma_{\mu}}{m}\right) .
$$

Moreover,

$$
\mathbb{L}_{k}^{-1}=\mathbb{L}_{k^{\pi}}, \quad k^{\pi \mu}=\left(k^{0},-\mathbf{k}\right) .
$$

To determine amplitudes $v(k)$ and $u(k)$, we use the Weinberg conditions (8). The most general form of $v(k)$ fulfilling $(8)$ reads

$$
v(k)=\left(\begin{array}{l}
c_{1} \mathbb{L}_{k} \\
c_{2} \mathbb{L}_{k}^{-1}
\end{array}\right), \quad c_{1}, c_{2} \in \mathbb{C},
$$

where $\mathbb{L}_{k}$ is given in (80). Now, the Dirac equation (4a) implies that

$$
c_{2}=-c_{1}
$$

Further restrictions on $c_{1}$ come from the normalization condition. Requiring that $v(k) \bar{v}(k)=-\Lambda_{-}$(the standard projector defined in (5)), we obtain $c_{1}=\frac{\chi}{\sqrt{2}}$ with $|\chi|=1$. Under this choice, also relation (84) holds. Since the phase factor $\chi$ is irrelevant, we set for convenience $\chi=1$. In this way, we arrive at formula (12).

Here we gathered some useful relations for the amplitudes $v(k)$ and $u(k)$ :

$$
\begin{aligned}
& \bar{v}(k) v(k)=-I_{2}, \\
& v(k) \bar{v}(k)=\frac{k \gamma-m I}{2 m}=-\Lambda_{-}, \\
& \bar{u}(k) u(k)=I_{2}, \\
& u(k) \bar{u}(k)=\frac{k \gamma+m I}{2 m}=\Lambda_{+} .
\end{aligned}
$$

\section{References}

1. Alsing, P.M., Milburn, G.J.: Lorentz invariance of entanglement. Quantum Inf. Comput. 2, 487-512 (2002)

2. Bartlett, S.D., Terno, D.R.: Relativistically invariant quantum information. Phys. Rev. A 71, 012,302 (2005)

3. Barut, A.O., Rączka, R.: Theory of Group Representations and Applications. PWN, Warszawa (1977)

4. Bauke, H., Ahrens, S., Keitel, C.H., Grobe, R.: What is the relativistic spin operator? New J. Phys. 16, 043,012 (2014)

5. Bittencourt, V.A.S.V., Bernardini, A.E., Blasone, M.: Global Dirac bispinor entanglement under Lorentz boosts. Phys. Rev. A 97, 032,106 (2018)

6. Bliokh, K.Y., Dennis, M.R., Nori, F.: Position, spin, and orbital angular momentum of a relativistic electron. Phys. Rev. A 96, 023,622 (2017)

7. Bradler, K.: Relativistically invariant photonic wave packets. J. Opt. Soc. Am. B 28, 727-736 (2011) 
8. Bradler, K., Castro-Ruiz, E., Nahmad-Achar, E.: Quantum and classical capacity boosted by a Lorentz transformation. Phys. Rev. A 90, 022,308 (2014)

9. Caban, P., Dziȩgielewska, A., Karmazyn, A., Okrasa, M.: Polarization correlations of Dirac particles. Phys. Rev. A 81, 032,112 (2010)

10. Caban, P., Rembieliński, J.: Photon polarization and Wigner's little group. Phys. Rev. A 68, 042,107 (2003)

11. Caban, P., Rembieliński, J.: Lorentz-covariant reduced spin density matrix and Einstein-PodolskyRosen-Bohm correlations. Phys. Rev. A 72, 012,103 (2005)

12. Caban, P., Rembieliński, J.: Einstein-Podolsky-Rosen correlations of Dirac particles: quantum field theory approach. Phys. Rev. A 74, 042,103 (2006)

13. Caban, P., Rembieliński, J., Włodarczyk, M.: Strange bahavior of the relativistic Einstein-PodolskyRosen correlations. Phys. Rev. A 79, 014,102 (2009)

14. Caban, P., Rembieliński, J., Włodarczyk, M.: Spin operator in the Dirac theory. Phys. Rev. A 88, 022,119 (2013)

15. Czachor, M.: Einstein-Podolsky-Rosen-Bohm experiment with relativistic massive particles. Phys. Rev. A 55, 72-77 (1997)

16. Czachor, M.: Comment on 'Quantum entropy and special rlativity' by A. Peres, P. F. Scudo, and D. R. Terno, Phys. Rev. Lett. 88, 230402 (2002). Phys. Rev. Lett. 94, 078,901 (2005)

17. Friedman, Y., Scarr, T.: Covariant uniform acceleration. J. Phys.: Conf. Ser. 437, 012,009 (2013)

18. Friedman, Y., Scarr, T.: Spacetime transformations from a uniformly accelerated frame. Phys. Scr. 87, 055,004 (2013)

19. Lamata, L., Martin-Delgado, M.A., Solano, E.: Relativity and Lorentz invariance of entanglement distillability. Phys. Rev. Lett. 97, 250,502 (2006)

20. Mashhoon, B., Muench, U.: Length measurement in accelerated systems. Ann. Phys. (Leipzig) 11, 532-547 (2002)

21. Omkar, S., Banerjee, S., Srikanth, R., Alok, A.K.: The Unruh effect interpreted as a quantum noise channel. Quantum Inf. Comput. 16, 757-770 (2016)

22. Peres, A., Scudo, P.F., Terno, D.R.: Quantum entropy and special relativity. Phys. Rev. Lett. 88, 230,402 (2002)

23. Peres, A., Terno, D.R.: Quantum information and relativity theory. Rev. Mod. Phys. 76, 93-124 (2004)

24. Rembieliński, J., Smoliński, K.A.: Einstein-Podolsky-Rosen correlations of spin measurements in two moving inertial frames. Phys. Rev. A 66, 052,114 (2002)

25. Saldanha, P.L., Vedral, V.: Physical interpretation of the Wigner rotations and its implications for relativistic quantum information. New J. Phys. 14, 023,041 (2012)

26. Saldanha, P.L., Vedral, V.: Spin quantum correlations of relativistic particles. Phys. Rev. A 85, 062,101 (2012)

27. Saldanha, P.L., Vedral, V.: Wigner rotations and an apparent paradox in relativistic quantum information. Phys. Rev. A 87, 042,102 (2013) 